\title{
Harmonic Analyses with Measuring Electromagnetic Forces and Torques for Magnetically Levitated Planar Motors
}

\author{
Shengguo Zhang ${ }^{1, a^{*}}$, Shuo Zhang ${ }^{2, b}$, and Wanchun Liu ${ }^{1, c}$ \\ ${ }^{1}$ School of Electrical Engineering, Northwest University for Nationalities, Lanzhou, 730030, China \\ ${ }^{2}$ School of Automation, Hangzhou Dianzi University, Hangzhou, 310018, China

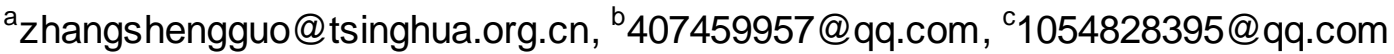

Keywords: Harmonics, Frequency spectrum amplitude, Power spectral density, Electromagnetic forces/torques.

Abstract: This paper focuses on analyzing the harmonics of electromagnetic forces and torques of a magnetically levitated planar motor by measuring these forces and torques. By a multi-dimension force/torque sensor measure system, all electromagnetic forces and torques of a planar motor are measured simultaneously. And then these measured data are processed and analyzed in terms of frequency orders, frequency spectrum amplitudes, and power spectral densities. Data analyses indicate the real electromagnetic forces/torques include different odd order harmonics which result from the Halbach magnetic field. The frequency spectrum amplitudes of harmonics attenuate with the increasing of the harmonic orders and the power spectral densities of harmonics diminish fast with the increasing of the harmonic orders. These harmonic analyses can be used to select control strategy and closed-loop bandwidth for the real-time control of the magnetically levitated planar motor.

\section{Introduction}

Magnetically levitated planar motors (MLPM) have been developed in recent years, as alternatives to air bearing stages constructed of stacked linear motors. These motors usually consist of two parts, stator and mover, and they can be constructed in two ways. One way is that the mover consists of coil array and the stator of permanent magnet array (MLPM with moving coils) [1,2]. The other way is that the mover consists of permanent magnet array and the stator of coil array (MLPM with moving magnets) [3, 4]. No matter in which way they are constructed, it is very important to understand the electromagnetic forces/torques so as to obtain better motion control performances.

In literature [3-5], the electromagnetic forces/torques of MLPM with moving magnets have been modeled and motors have been controlled. While the electromagnetic forces/torques model is used to commutate and control the planar motor, it has to be simplified as an analytical model which only includes the $1^{\text {st }}$ order harmonic. This challenges the control implementation seriously. In literature [6], the force and back-EMF constant expressions of a MLPM with moving coils were derived and tested, yet the torque expressions were not included. References [7, 8] also showed the modeland the control of a MLPM with moving coils. Due to the model error of electromagnetic forces/torques, however, there has not attained good control performances and positioning accuracy.

In this paper, harmonics of electromagnetic forces/torques are analyzed based on comprehensive measurements of electromagnetic forces/torques of a MLPM with moving coils. Firstly, the planar motor under investigation is presented briefly, the potential harmonics of real electromagnetic forces/torques are analyzed, and the electromagnetic force/torque measurement system and scheme are introduced. And then, the measured data are processed and analyzed in frequency domain so as to give deeper insight into the harmonics of real electromagnetic forces and torques. 


\section{Magnetically Levitated Planar Motor and Its Electromagnetic Force/Torque Harmonics}

Configuration of Magnetically Levitated Planar Motor under Investigation. The planar motor under investigation is designed to have a large area stator and a long travel mover. Its stator is a Halbach permanent magnet array (HPMA), and its mover contains 20 ironless coils arranged in certain order. Fig. 1 (a) and (b) show the coil array mover and the permanent magnet array stator (partial), respectively.

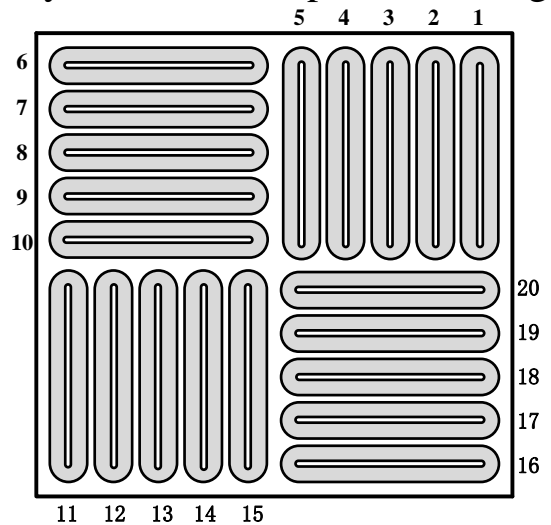

(a)

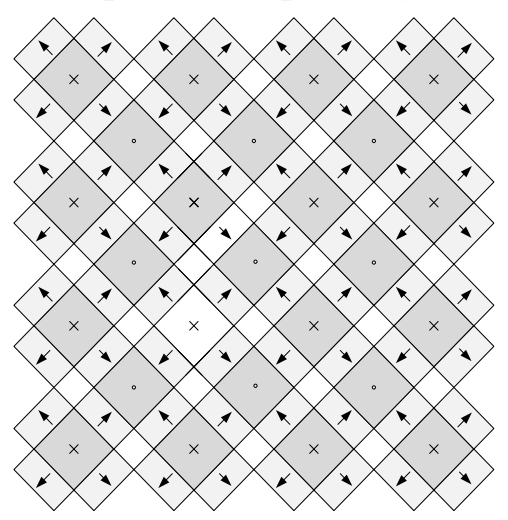

(b)

Fig. 1 Investigated magnetically levitated planar motor: (a) Bottom view of mover; (b) Top view of stator (partial)

Given that one coil is commutated unit current, $1 \mathrm{~A}$, the $x$-, $y$-direction propulsion force $F_{x}, F_{y}, z$-direction levitation force $F_{z}$, and $x$-, $y$-, $z$-axis torques $T_{x}, T_{y}, T_{z}$, which act on the mover of the motor or generate by this coil in Halbach permanent magnet field, can all be integrated by using volume integral of Lorenz force law $[7,8]$. They are denoted by force/torque vector $\boldsymbol{W r}(\boldsymbol{q})$ :

$$
\boldsymbol{W r}(\boldsymbol{q})=\left[F_{x}, F_{y}, F_{z}, T_{x}, T_{y}, T_{z}\right]^{\mathrm{T}} .
$$

For example, $z$-direction levitation force $F_{z}$, can be calculated as:

$$
\begin{aligned}
F_{z} & =\int_{V} \mathrm{~J} \times B_{m X} d V \\
& =\frac{2 \sqrt{2} N_{c} l_{c} B_{x y} \tau^{2}}{\pi^{2} b_{c}} \sin \left(\frac{\pi w_{c}}{2 \tau}\right) \sin \left(\frac{\pi b_{c}}{2 \tau}\right) \exp \left(-\frac{\pi h_{c e}}{\tau}\right) \cos \left[\frac{\pi}{\tau}\left(x+x_{c}\right)\right],
\end{aligned}
$$

where $B_{x y}$ is the amplitude of the $1^{\text {st }}$ order harmonic of the magnetic flux density distribution at $z=0$ and $\tau$ is the magnetic pole pitch. The equivalent sizes of coil, length, width, and width of turn are denoted by $l_{c}, w_{c}$, and $b_{c}$ respectively. $N_{c}$ is the number of coil turns and $h_{c e}$ is equivalent thickness of real coil thickness $h_{c}$. And $x$ is mover position relative to stator in $x$-direction; $x_{c}$ is coil $x$-direction position in mover.

Harmonic Estimation. Under conditions that sizes of coil are determined and gap between coil array and HPMA is invariant, Eq. 2 can be rewritten as:

$$
F_{z}=\mathrm{F}_{z m} \cos \left[\frac{\pi}{\tau}\left(x+x_{c}\right)\right],
$$

where $\mathrm{F}_{z m}$ is the amplitude of $F_{z}$. Assuming the relative moving velocity of coil array is a constant $\mathrm{v}$ at any time $t$, it yields:

$$
F_{z}=\mathrm{F}_{z m} \cos \left[\frac{\pi}{\tau}\left(\mathrm{v} t+x_{c}\right)\right]
$$

As can be seen from Eq. $4, F_{z}$ is the cosine function of $x$. The frequency of this cosine function is:

$$
\mathrm{f}_{1}=\frac{\pi \mathrm{v}}{\tau} \frac{1}{2 \pi}=\frac{\mathrm{v}}{2 \tau}
$$

The electromagnetic forces and torques are decided by both the magnetic field and the coil current. When the coil current is constant, $1 \mathrm{~A}$, the electromagnetic forces and torques are decided only by the magnetic field. 
So frequency $f_{1}$ of electromagnetic forces/torques originates from the $1^{\text {st }}$ order harmonic of magnetic field. In light of this way, frequencies of different order harmonics of electromagnetic forces/torques can be inferred:

$$
\mathrm{f}_{\mathrm{n}}=\mathrm{nf}_{1}=\frac{\mathrm{nv}}{2 \tau}, \mathrm{n}=1,2,3, \cdots
$$

Eq. 6 can also be inferred from representations of other electromagnetic forces/torques, $F_{x}, F_{y}, T_{x}, T_{y}$, and $T_{z}$. Therefore, once real electromagnetic forces/torques are measured at enough frequency, it is possible to give deeper insight into the higher order harmonics of real electromagnetic forces/torques.

Multi-Dimension Force/Torque Measurement System and Measurement Scheme. In order to measure the real electromagnetic forces/torques, a multi-dimension force/torque measurement system is set up. Fig. 2 (a) and (b) show the photograph and the configuration of the measurement system, respectively. The multi-dimension force/torque sensor is mounted on a beam via an upper mounting rack. It can measure three force components and three torque components in three-dimension Euclid space, simultaneously.

The coil array is connected with the sensor via the lower mounting rack. An adjustable mechanism adjusts the sensor's height so that the gap between coil and HPMA can be changed. The HPMA stator is mounted on the $X-Y$ plane motion mechanism which is driven by two controlled step motors. So the HPMA stator can move in desired acceleration, velocity, and displacement in horizontal plane. All measurements are finished under conditions of constant gap between coil array and HPMA, $2 \mathrm{~mm}$, constant velocity, 10mm/s, and constant current, 1A. For eliminating the disturbance of coil cable, the HPMA stator instead of the coil array is moving relatively and the coil array and the sensor are always stationary. This does not change the relative motion relationship between coil array and HPMA.

Data measured by the sensor are preprocessed and then transmitted to the host computer. Data sampling frequency of the measurement system is at $250 \mathrm{~Hz}$.

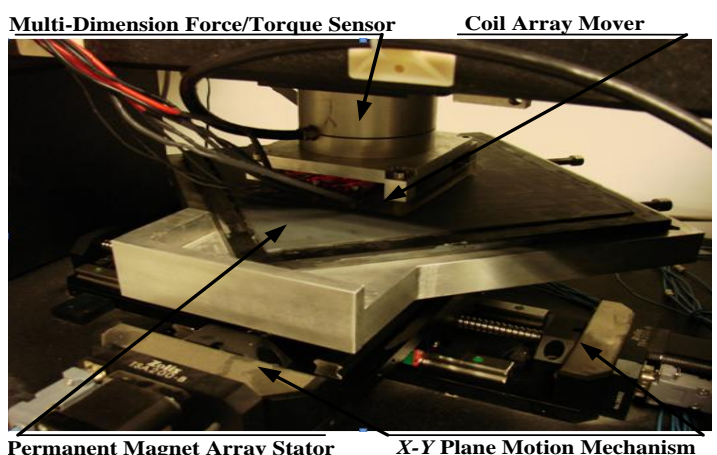

(a)

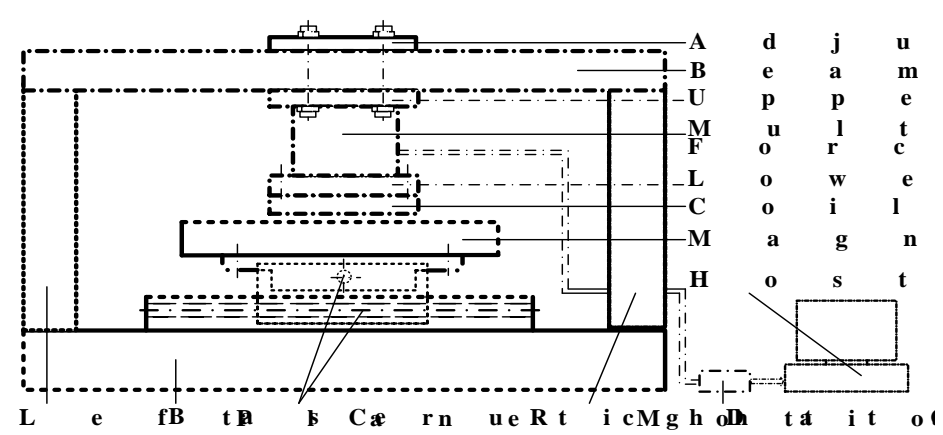

(b)

Fig. 2 Multi-dimension force/torque measurement system: (a) Photograph; (b) Configuration

\section{Harmonic Analyses of Electromagnetic Forces and Torques}

According to above mentioned measurement scheme, measurements are performed many times in different movement routes. Fig. 3 (a) and (b) show the data of one of many measurements.

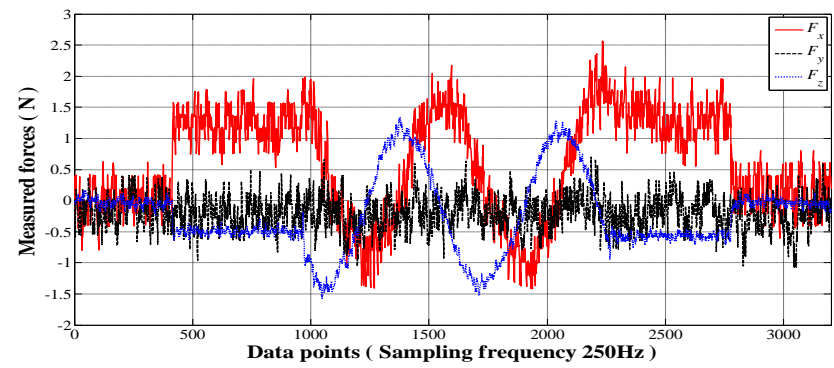

(a)

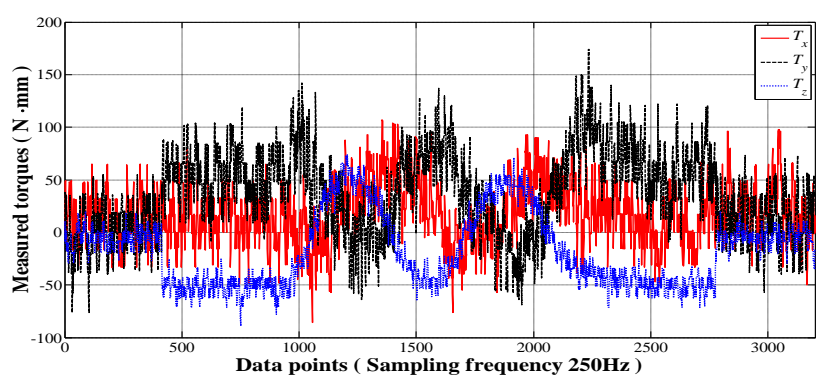

(b)

Fig. 3 Measured data of electromagnetic forces/torques: (a) $F_{x}, F_{y}$, and $F_{z}$; (b) $T_{x}, T_{y}$, and $T_{z}$ 
FromFig. 3 it can be observed that, all effective data of electromagnetic forces/torques are in approximate sinusoidal/cosinusoidal distribution except for $F_{y}$ to be about zero. In addition, the sinusoida/cosinusoidal waves are mixed with lots of ripples. Fig. 4 (a) and (b) show the discrete frequency spectrum amplitudes of measured electromagnetic forces/torques, respectively.

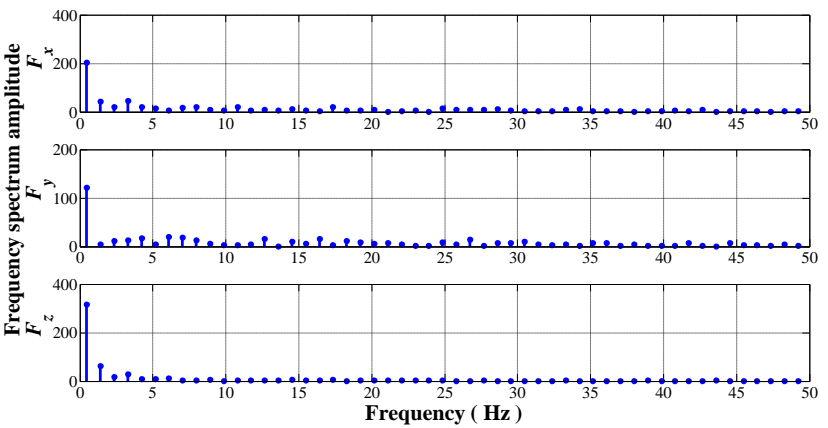

(a)

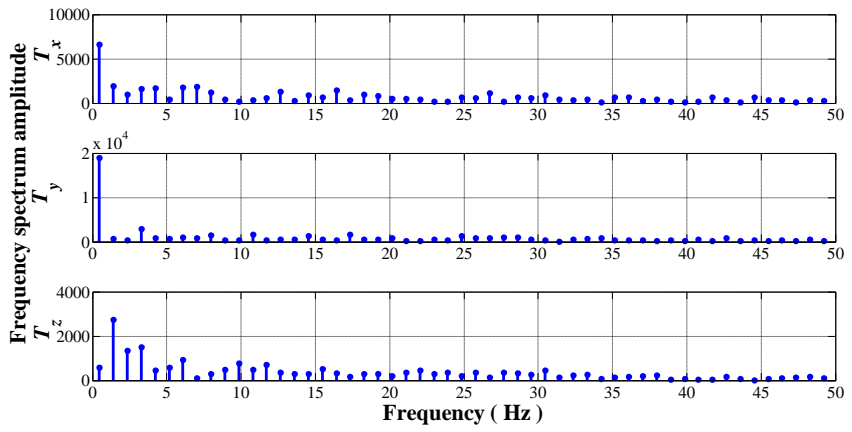

(b)

Fig. 4Frequency spectrum amplitudes of measured electromagnetic forces/torques: (a) $F_{x}, F_{y}$, and $F_{z} ;$ (b) $T_{x}, T_{y}$, and $T_{z}$

In order to eliminate the measuring noises, a low-pass filter, whose cutoff frequency is at $10 \mathrm{~Hz}$, is designed to filter the measured data [9]. Fig. 5 (a) and (b) show measured results of electromagnetic forces/torques after low-pass filtering, respectively. Fig. 6 (a) and (b) show the discrete frequency spectrum amplitudes of measured electromagnetic forces/torques after filtering, respectively.

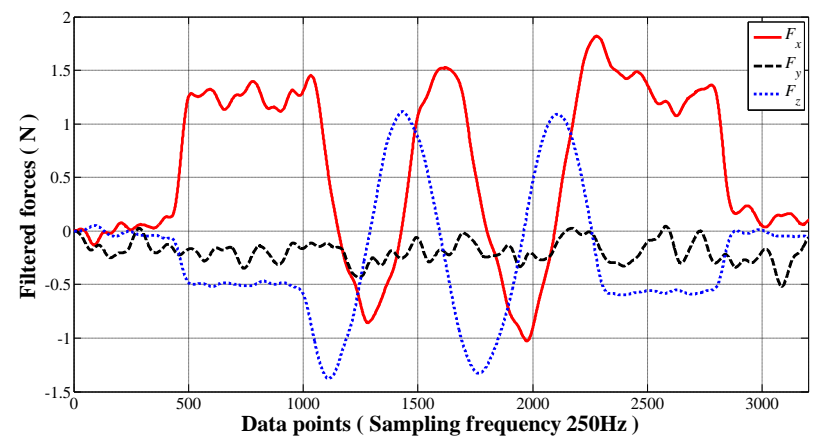

(a)

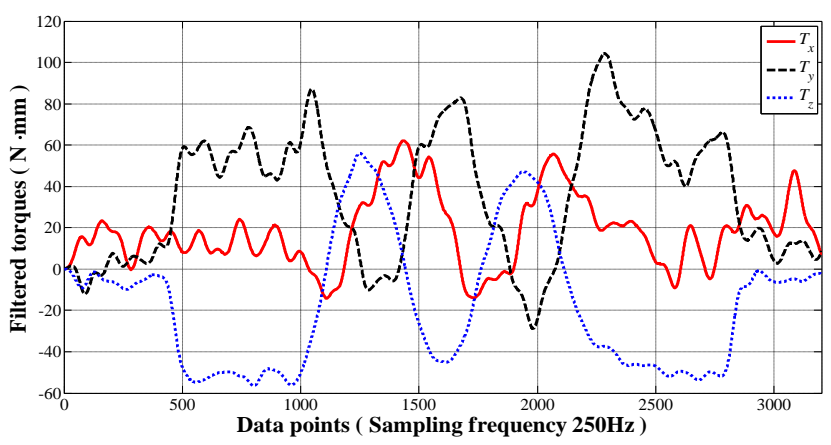

(b)

Fig. 5 Measured data of measured electromagnetic forces/ torques after filtering: (a) $F_{x}, F_{y}$, and $F_{z}$; (b) $T_{x}, T_{y}$, and $T_{z}$

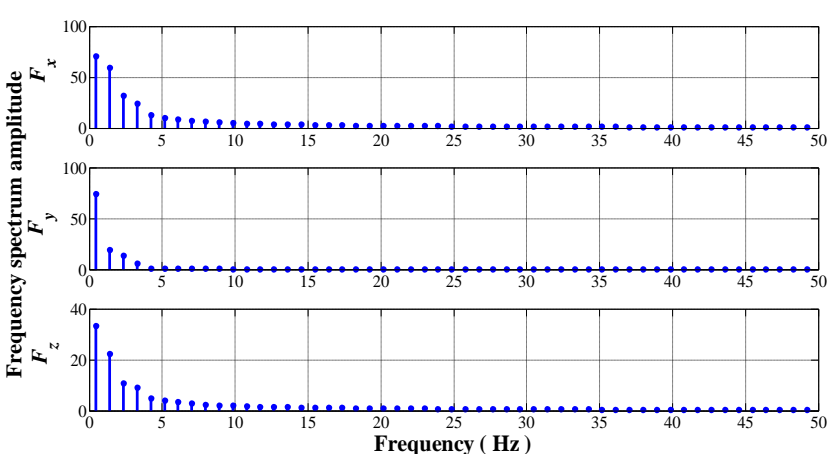

(a)

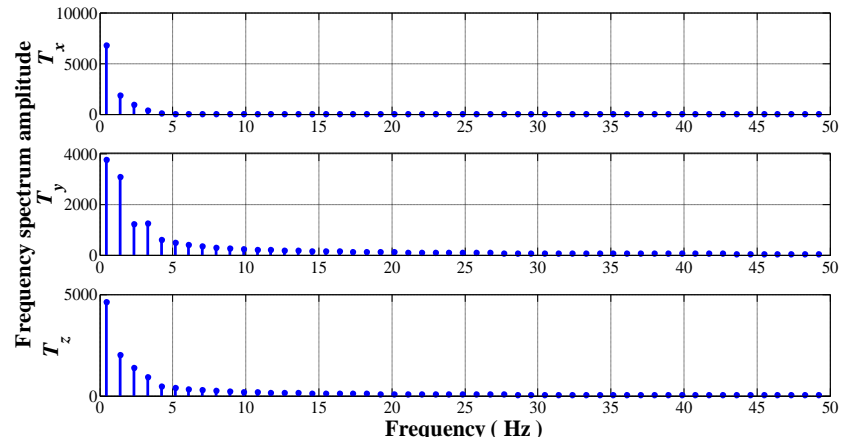

(b)

Fig. 6Frequency spectrum amplitudes of measured forces/ torques after filtering: (a) $F_{x}, F_{y}$, and $F_{z}$; (b) $T_{x}, T_{y}$, and $T_{z}$

According to Eq. 6, due to the relative motion velocity between coil array and HPMA is: $v=10 \mathrm{~mm} / \mathrm{s}$ and the nominal magnetic pole pitch is: $\tau=10.6066 \mathrm{~mm}$, the theoretical $1^{\text {st }}$ order harmonic frequency should be: $\mathrm{f}_{1}$ $=\mathrm{v} /(2 \tau)=0.4717 \mathrm{~Hz}$. Yet from Fig. 6 there can be observed that the real $1^{\text {st }}$ order harmonic frequency is $0.4688 \mathrm{~Hz}$. Two frequencies are approximately equal. This indicates that the frequency of $0.4688 \mathrm{~Hz}$ is just 
the real frequency of the $1^{\text {st }}$ order harmonic of electromagnetic forces/torques. In other words, because the real frequency of the $1^{\text {st }}$ order harmonic is $0.4688 \mathrm{~Hz}$, the real magnetic pole pitch should be: $\tau=\mathrm{v} /\left(2 \mathrm{f}_{1}\right)=$ $10.46655 \mathrm{~mm}$. It indicates the real magnetic pole pitch becomes longer slightly after the HPMA is fabricated.

Correspondingly, the real frequencies of other higher order harmonics observed from Fig. 6 can also be compared with the theoretical frequencies of different order harmonics calculated from Eq. 6 . Table 1 shows the real and theoretical frequencies of different order harmonics.

Table 1 Theoretical frequencies and real frequencies of different order harmonics (within the $11^{\text {th }}$ order harmonics)

\begin{tabular}{ccccccc}
\hline Harmonic orders & $1^{\text {st }}$ order & $3^{\text {rd }}$ order & $5^{\text {th }}$ order & $7^{\text {th }}$ order & $9^{\text {th }}$ order & $11^{\text {th }}$ order \\
\hline Theoretical values & 0.4717 & 1.4151 & 2.3585 & 3.3019 & 4.2453 & 5.1887 \\
\hline Real values & 0.4688 & 1.4063 & 2.3438 & 3.2813 & 4.2188 & 5.1563 \\
\hline
\end{tabular}

More observations from Fig. 6 indicate, for the different order harmonics, their frequency spectrum amplitudes decay with the harmonic orders. Table 2 shows the normalized frequency spectrum amplitudes within the $11^{\text {th }}$ order harmonics. Beyond the $11^{\text {th }}$ order harmonic, their amplitudes attenuate to be very small amount compared with the amplitude of the $1^{\text {st }}$ order harmonic.

Table 2 Normalized frequency spectrum amplitudes of different order harmonics (within the $11^{\text {th }}$ order harmonics)

\begin{tabular}{ccccccc}
\hline Harmonics orders & $1^{\text {st }}$ order & $3^{\text {rd }}$ order & $5^{\text {th }}$ order & $7^{\text {th }}$ order & $9^{\text {th }}$ order & $11^{\text {th }}$ order \\
\hline$F_{x}$ & 1 & 0.8447 & 0.4594 & 0.3471 & 0.1880 & 0.1492 \\
\hline$F_{y}$ & 1 & 0.2590 & 0.1807 & 0.0777 & 0.0174 & 0.0171 \\
\hline$F_{z}$ & 1 & 0.6752 & 0.3240 & 0.2770 & 0.1487 & 0.1202 \\
\hline$T_{x}$ & 1 & 0.2716 & 0.1416 & 0.0612 & 0.0132 & 0.0087 \\
\hline$T_{y}$ & 1 & 0.8581 & 0.3438 & 0.3507 & 0.1702 & 0.1356 \\
\hline$T_{z}$ & 1 & 0.4346 & 0.3031 & 0.2027 & 0.1023 & 0.0876 \\
\hline Mean & 1 & 0.5572 & 0.2921 & 0.2194 & 0.1066 & 0.0854 \\
\hline
\end{tabular}

Furthermore, the power spectrums of different order harmonics are analyzed by using Burg algorithm based on AR (auto-regressive) model [10]. Fig. 7 (a) and (b) show the discrete power spectral densities of measured electromagnetic forces/torques after filtering, respectively.

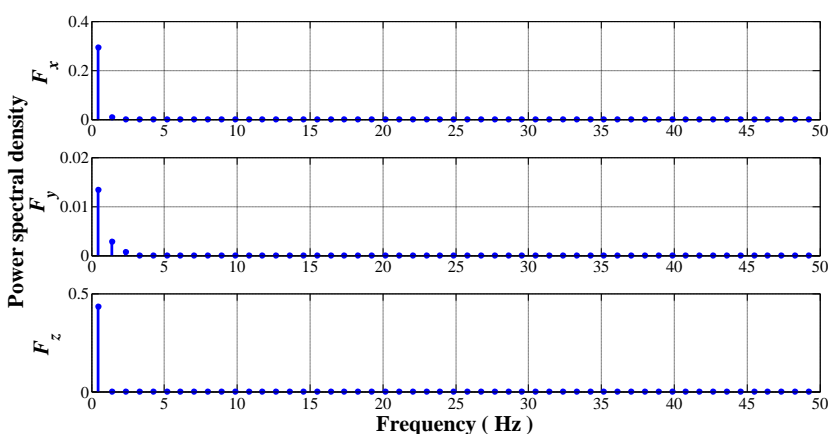

(a)

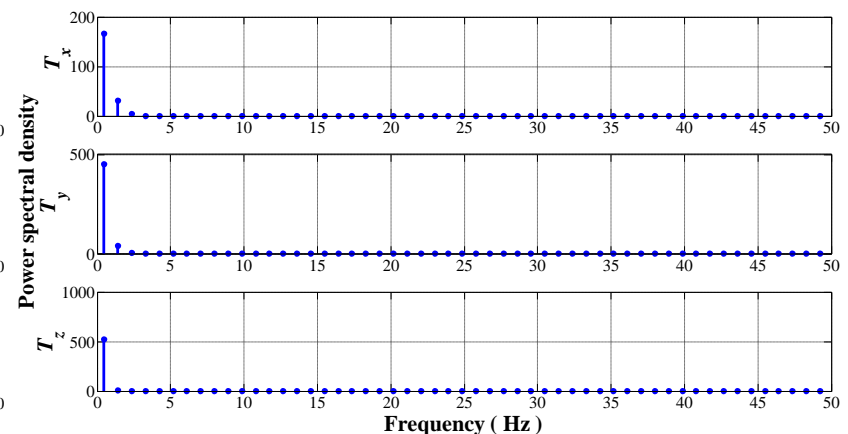

(b)

Fig. 7 Power spectral densities of measured forces/torques after filtering: (a) $F_{x}, F_{y}$, and $F_{z}$; (b) $T_{x}, T_{y}$, and $T_{z}$

As can be observed from Fig. 7 , the frequency spectral densities of different order harmonics fast attenuate with the harmonic orders. Table 3 shows the normalized power spectral densities within the $9^{\text {th }}$ order harmonics. After the $9^{\text {th }}$ order harmonic, their power spectral densities diminish to be nearly zeros compared with the power spectral density of the $1^{\text {st }}$ order harmonic.

Fromabove harmonic analyses, electromagnetic forces and torques include not only the $1^{\text {st }}$ order harmonic components but also other higher order harmonic components. These higher order harmonic components may disturb the control of MLPM. In order to suppress these disturbances from higher order harmonics of real electromagnetic forces/torques, a compromise bandwidth of closed-loop system, which had better be lower than the frequency of the $3^{\text {rd }}$ order harmonic of real electromagnetic forces/torques, should be chosen 
in control system design. For example, the closed-loop bandwidth should be less than $\mathrm{f}_{3}=3 \mathrm{v} /(2 \tau)=1.5 \mathrm{v} / \tau \mathrm{Hz}$, in accordance with Eq.6.

Table 3 Normalized Power spectral densities of different order harmonics (within the $9^{\text {th }}$ order harmonics)

\begin{tabular}{cccccc}
\hline Harmonics orders & $1^{\text {st }}$ order & $3^{\text {rd }}$ order & $5^{\text {th }}$ order & $7^{\text {th }}$ order & $9^{\text {th }}$ order \\
\hline$F_{x}$ & 1 & 0.0289 & 0.0017 & 0.0002 & $<0.0001$ \\
\hline$F_{y}$ & 1 & 0.2222 & 0.0593 & 0.0059 & $<0.0005$ \\
\hline$F_{z}$ & 1 & 0.0021 & 0.0001 & 0.00002 & $<0.00001$ \\
\hline$T_{x}$ & 1 & 0.1900 & 0.0288 & 0.0032 & $<0.0003$ \\
\hline$T_{y}$ & 1 & 0.0902 & 0.0085 & 0.0012 & $<0.0001$ \\
\hline$T_{z}$ & 1 & 0.0154 & 0.0009 & 0.0001 & $<0.00001$ \\
\hline Mean & 1 & 0.0915 & 0.0166 & 0.0018 & $<0.00017$ \\
\hline
\end{tabular}

\section{Conclusions}

The actual electromagnetic forces/torques of a MLPM with moving coils are measured simultaneously on a multi-dimension force/torque sensor measurement system. And then the measured data are processed and analyzed in terms of frequency order, frequency spectrum amplitude, and power spectral density. These analyses illustrate the electromagnetic forces/torques include different odd order harmonics, the frequency spectrum amplitudes of these harmonics decay with the increasing of the harmonic orders, and the power spectral densities of these harmonics quickly decrease with the increasing of the harmonic orders. These harmonic characteristics can be used to design the closed-loop control system of the magnetically levitated planar motor.

\section{Acknowledgements}

This work was financially supported by National Natural Science Foundation of China (51465053) and Fundamental Research Funds for the Central Universities (31920140082).

\section{References}

[1] J. C. Compter, Electro-dynamic planar motor, Precis. Eng., 28 (2) 171-180, 2004.

[2] Y. Zhu, F. Liu, M. Zhang, et al., Optimal design of ironless permanent magnet planar motor for minimisation of force ripples, IET Electr. Power App., 7 (4) 321-330, 2013.

[3] J. W. Jansen, C. M. M. Lierop, E. A. Lomonova, et al., Modelling of magnetically levitated planar actuators with moving magnets, IEEE T. Magn., 43 (1) 15-25, 2007.

[4] J. M. M. Rovers, J. W. Jansen, J. C. Compter, et al., Modelling of magnetically levitated planar actuators with moving magnets, IEEE T. Ind. Electron., 59 (5) 2157-2166, 2012.

[5] C. M. M. Lierop, J. W. Jansen, A. A. H. Damen, et al., Model-based commutation of a long-stroke magnetically levitated linear actuators, IEEE T. Ind. Appl., 45 (6) 1982-1990, 2009.

[6] H. S. Cho, H. K Jung, Analysis and design of synchronous permanent magnet planar motors, IEEE T. Energy Conver., 17 (4) 492-499, 2002.

[7] S. G. Zhang, X. P. Dang, K. Wang, Modelling of electromagnetic force/torque for magnetically levitated planar motor, in Proc. Int. Conf. Mechatronics, Robotics and Automation 311-316, 2013.

[8] S. G. Zhang, Y. Zhu, H. H. Mu, et al., Decoupling and levitation control of a six-degree-of-freedom magnetically levitated stage with moving coils based on commutation of coil array, P. I. Mech. Eng. I-J. Syst., 226 (7) 875-886, 2012. 
[9] R. Chassaing, D. Reay, Digital signal processing and applications with the TMS320C6713 and TMS320C6416 DSK, Hoboken: John Wiley \& Sons, the 2nd ed., 2008.

[10] G. S. Hu, Digital signal processing: theory, algorithm, and implementation, Tsinghua University Press, the 2nd ed., 551-554,571-577, 2003. 